\title{
Biological Factors Contributing to the Response to Cognitive Training in Mild Cognitive Impairment
}

\author{
Jessica Peter $^{\mathrm{a}, \mathrm{b}, *}$, Lena V. Schumacher ${ }^{\mathrm{c}}$, Verena Landerer ${ }^{\mathrm{b}}$, Ahmed Abdulkadir ${ }^{\mathrm{a}, \mathrm{d}}$, \\ Christoph P. Kallere,f , Jacob Lahr ${ }^{\mathrm{b}}$ and Stefan Klöppel ${ }^{\mathrm{a}, \mathrm{g}}$ \\ ${ }^{a}$ University Hospital of Old Age Psychiatry and Psychotherapy, University of Bern, Switzerland \\ ${ }^{\mathrm{b}}$ Department of Psychiatry and Psychotherapy, Faculty of Medicine, University of Freiburg, Germany \\ ${ }^{\mathrm{c}}$ Medical Psychology and Medical Sociology, Faculty of Medicine, University of Freiburg, Germany \\ ${ }^{\mathrm{d}}$ Department of Computer Science, University of Freiburg, Germany \\ ${ }^{\mathrm{e}}$ Department of Neurology, Faculty of Medicine, University of Freiburg, Germany \\ ${ }^{\mathrm{f}}$ BrainLinks-BrainTools Cluster of Excellence, University of Freiburg, Freiburg, Germany \\ ${ }^{\mathrm{g}}$ Centre for Geriatric Medicine and Gerontology, Faculty of Medicine, University of Freiburg, Germany
}

Accepted 11 September 2017

\begin{abstract}
In mild cognitive impairment (MCI), small benefits from cognitive training were observed for memory functions but there appears to be great variability in the response to treatment. Our study aimed to improve the characterization and selection of those participants who will benefit from cognitive intervention. We evaluated the predictive value of diseasespecific biological factors for the outcome after cognitive training in MCI $(n=25)$ and also considered motivation of the participants. We compared the results of the cognitive intervention group with two independent control groups of MCI patients (local memory clinic, $n=20$; ADNI cohort, $n=302$ ). The primary outcome measure was episodic memory as measured by verbal delayed recall of a 10-word list. Episodic memory remained stable after treatment and slightly increased 6 months after the intervention. In contrast, in MCI patients who did not receive an intervention, episodic memory significantly decreased during the same time interval. A larger left entorhinal cortex predicted more improvement in episodic memory after treatment and so did higher levels of motivation. Adding disease-specific biological factors significantly improved the prediction of training-related change compared to a model based simply on age and baseline performance. Bootstrapping with resampling ( $n=1000)$ verified the stability of our finding. Cognitive training might be particularly helpful in individuals with a bigger left entorhinal cortex as individuals who did not benefit from intervention showed $17 \%$ less volume in this area. When extended to alternative treatment options, stratification based on disease-specific biological factors is a useful step towards individualized medicine.
\end{abstract}

Keywords: Cognitive training, entorhinal cortex, episodic memory, mild cognitive impairment, response prediction

\section{INTRODUCTION}

In mild cognitive impairment (MCI), the pre-dementia stage of Alzheimer's disease (AD), pharmacological treatment is ineffective, as acetylcholinesterase inhibitors fail to reduce incident dementia [1, 2] and to improve cognition [3].

\footnotetext{
${ }^{*}$ Correspondence to: Jessica Peter, PhD, University of Old Age Psychiatry and Psychotherapy, Bolligenstrasse 111, 3000 Bern 60, Germany. Tel.: +49 03193289 03; E-mail: jessica.peter@puk. unibe.ch.
}

Non-pharmacological therapies such as cognitive interventions are thus gaining importance. Cognitive interventions can be divided into cognitive stimulation, cognitive rehabilitation, and cognitive training [4]. Among those, cognitive training, which focuses on guided practice on a set of tasks that reflect particular cognitive functions, is beneficial in MCI [5, 6]. Cognitive training can be divided into strategy training (i.e., the instruction and practice of mnemonic approaches such as the method of loci or visual imagery) and repetitive, often 
computer-based, cognitive exercises [7]. Cognitive exercises tend to benefit the specific training task or domain (but see Noack et al. [8]), while strategy training enhances cognitive functions beyond that [7]. Cognitive interventions are typically offered for 5-8 weeks; longer training programs did not consistently show better results [5].

In MCI, benefits from strategy training were observed for verbal episodic memory (i.e., verbal delayed recall) and the Mini-Mental Status Examination (MMSE) [6, 9]. Two studies found strategy training to stabilize verbal delayed recall over three or six months, respectively, despite a progressing neurodegenerative disorder $[10,11]$. An additional study $(n=15)$ reported improved processing speed in MCI, when combining strategy training with psychoeducation; the effects were maintained after 6-months follow-up [12] and even for up to 28 months for the MMSE [13]. Thus, strategy training seems to be promising in prodromal stages of $\mathrm{AD}$, providing the possibility to offer an early intervention.

However, there appears to be great variability in the response to cognitive training among participants, although it has been rarely addressed explicitly. The combined training study by Londos and colleagues [12] for example, found $47 \%$ improved, $40 \%$ remained unchanged, while $13 \%$ worsened in processing speed. In another study in individuals with subjective, but not objective, memory impairment (SMI, a population at increased risk of developing dementia; $n=19$ ), $21 \%$ worsened after cognitive training [14]. Most of the other studies have addressed response variability simply by reporting treatment effects in terms of average raw scores before and after training or by reporting the mean change and standard deviation, respectively.

Given that cognitive training is resource intensive, the characterization and selection of those participants who might benefit from treatment would greatly improve the practicability and effectiveness of those interventions. Yet, no study has explicitly aimed to predict the response to cognitive training in patients with either MCI or dementia due to AD and, thus, appropriate predictor variables for these populations are unknown. It seems obvious that motivation of the participants, but also social support, is a key element for a successful cognitive training. On the other hand, several intervention studies in healthy aging indicate a role of biological factors. For example, age and baseline cognitive status had an influence on the response to cognitive training in healthy elderly participants. Brooks et al. [15] found that older-old
( $\geq 70$ years of age) healthy participants $(n=286$ ) benefited more from strategy training than did younger-old ( $\leq 70$ years of age). In a study by Singer and colleagues $(n=96)$, perceptual speed at baseline was the best predictor of individual learning gains (in an episodic memory task) after strategy training in healthy elderly individuals [16]. In patients with MCI $(n=28)$, Belleville and colleagues found younger age to be significantly correlated with change in verbal episodic memory after cognitive training [17]. However, they also included healthy elderly controls $(n=17)$ and did not report if the correlation was significant in both groups. Apart from that, biological factors were only used as predictors for the outcome after medical treatment in MCI so far. Genetic variables (i.e., APOE) but neither volume of the hippocampus nor the basal forebrain predicted the response to donepezil (an acetylcholinesterase inhibitor) among those patients [18, 19]. However, larger left and right hippocampal volumes were predictive of less cognitive decline [18]. Concerning the response to cognitive training, the study by Engvig in individuals with SMI found that larger pre-training hippocampal volume was related to better verbal episodic memory one week after cognitive training [14]. Besides hippocampal volume, volume loss of the entorhinal cortex is an early indicator of pathological changes due to $\mathrm{AD}[20]$ and a strong predictor of prodromal AD in patients with MCI [21]. Furthermore, it is significantly correlated with delayed recall of word lists [22]. Thus, volume of the entorhinal cortex might serve as a biological predictor for the outcome after cognitive training, although not tested so far.

In the present study, we set out to evaluate the predictive value of biological factors when patients with MCI receive a six-week, well-established mnemonic strategy training [22]. Comparable to previous cognitive interventions studies (e.g., [23]), we did not evaluate the efficiency of that specific training but rather focused on predicting the response to intervention. Verbal episodic memory (i.e., verbal delayed recall) was used as the primary marker of the response to training, as it is typically [5] and effectively [6] targeted by strategy training in MCI. As both volume of the left hippocampus and the adjacent entorhinal cortex as well the presence of APOE $\varepsilon 4$ alleles are highly relevant for verbal learning [24-26], all three served as MRI-based or gene-based biological predictors, respectively. To study the transfer of mnemonic strategy training from the left hippocampus to the right hippocampus, we also assessed spatial 
navigation. Here, volume of right hippocampal subfields were used as MRI-based biological variables as spatial memory clearly involves the right hippocampus [27, 28]. Finally, to investigate more global cognitive changes, we also studied changes in speed of information processing which is independent of the hippocampal formation. For comparison to previous cognitive training studies in MCI (e.g., [13]), we also assessed the MMSE as a global marker of cognition despite its relatively low sensitivity to changes in the MCI stage [29]. Frequency of completed homework tasks indexed motivation.

\section{MATERIALS AND METHODS}

\section{Participants}

Twenty-five patients with amnestic MCI (73.4 \pm 5.3 years of age, range: $60-82 ; 13$ females; $13.3 \pm 3.3$ years of education, range: $8-20$ ) were included. The proportion of single-domain versus multiple-domain amnestic MCI was 7/18. All participants were recruited from the Center for Geriatric Medicine and Gerontology of the University Medical Center Freiburg. The study was approved by the Ethics committee of the University Medical Center Freiburg and was conducted according to the Declaration of Helsinki. All participants gave written informed consent prior to testing.

\section{Inclusion and exclusion criteria}

Cognitive functioning was evaluated using the Consortium to Establish a Registry of Alzheimer's disease (CERAD) neuropsychological battery [30] with German age-, gender-, and education-adjusted norms (http://www.memoryclinic.ch). Participants were diagnosed with MCI if at least one cognitive function was below 1.5 SD according to established criteria [31, 32]. Depressive symptoms were evaluated using Beck's Depression Inventory-II [33] and the Geriatric Depression Scale [34]. No participant reported a history of severe neurological or psychiatric disease, or drug or alcohol abuse. None of the participants was taking neuroactive drugs but low doses of sleep promoting medication did not lead to exclusion. We did not include patients with reported sudden steep decline of cognitive performance, focal neurological signs, major white matter changes (i.e., three points on the Fazekas scale [35]) to avoid cases with a predominantly vascular origin of cognitive changes.

\section{Genetic analyses}

The $\varepsilon 2 / 3 / 4$ haplotypes of the DNA samples were analyzed by polymerase chain reaction amplification of a 290-bp frame of exon 3 of the APOE gene and measurement of base mutations contained in codon 112 and 158 by dideoxy sequencing. The presence of APOE $\varepsilon 4$ alleles $(0,1$, or 2 ; coded as dummy variables) served in the prediction model detailed below.

\section{Study procedure}

At pre-training, all participants underwent baseline neuropsychological testing. Around 3-4 months after this baseline assessment ( $3.1 \pm 1.5$; range $1-5$ months), they received a six-week cognitive intervention (Fig. 1). Note that another neuropsychological assessment immediately before the intervention was avoided, to reduce the effects of training and habituation concerning the administered tasks. Participants underwent post-training neuropsychological assessment approximately one week ( \pm 2 days) after completion of the training $(6.2 \pm 2.8$ months after baseline; range 4-9 months). A final follow-up assessment was carried out after another interval of 6 months (Fig. 1). To reduce practice effects, an in-house parallel version of the CERAD wordlist was used in the assessment immediately after cognitive training, while at the final follow-up the initial wordlist was used again. Verbal delayed recall scores of the CERAD wordlist were used as a primary outcome measure of episodic memory. For speed of information processing, the Trail Making Test A (TMT-A, which is part of the CERAD) was used.

\section{Route Learning Task (RLT)}

To test navigation in a realistic scenario, we adapted a RLT which has been used in a previous study [36]. We did not use spatial memory from the CERAD two-dimensional spatial task (i.e., figure drawing and recall) as it is less prone to AD-related spatial impairment compared to real, three-dimensional navigation tasks [36]. The participants were taken on a route through the basement of the University Medical Centre Freiburg with only limited view of the outside environment (to inner courtyards). The route consisted of nine turns; all participants were tested in a wheelchair to account for differences in mobility. Although some of the participants had visited the clinic before, none had traversed the particular hallways of the test route. 


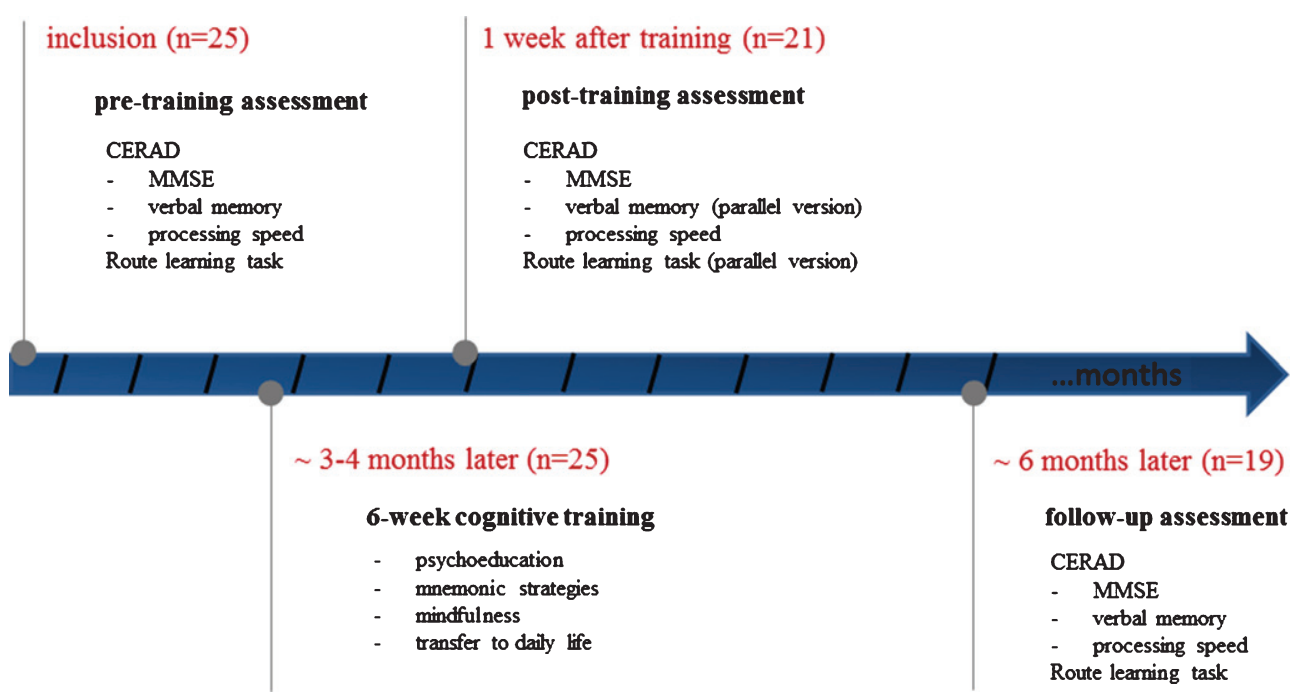

Fig. 1. Schematic of study procedure for patients with mild cognitive impairment receiving 6 weekly sessions of cognitive intervention and neuropsychological assessment at pre-training, post-training and follow-up. CERAD, Consortium to Establish a Registry of Alzheimer's disease; MMSE, Mini-Mental Status Examination.

Before testing, the participants were asked to pay attention to the route as they would be asked to repeat it themselves thereafter. After following the route once, the participants sat in the wheelchair again and at each corner of the route, they had to indicate directions to repeat the route forward (either verbally or by pointing). The experimenter noted each error and took the correct turn instead. After the cognitive intervention (i.e., at post-training), a parallel version of the initial RLT was done. At the final follow-up (12 months after baseline), the initial version of the RLT was used again.

\section{Cognitive intervention}

We offered a mnemonic strategy training in groups of 5-6 patients in six weekly sessions of $1.5 \mathrm{~h}$ each. Two out of three psychologists (JP, VL, LK), blinded to pre-training results, moderated the cognitive training using standardized documentation that adopted an well-established protocol [22]. In brief, the intervention consisted of psycho-educational information (1/2 session), of different mnemonics and strategies, including interactive imagery and face-name associations, to promote encoding and retrieval (5 sessions), and of mindfulness training plus information on the transfer of the strategies into daily life ( $1 / 2$ session) (see Supplementary Table 1 for more information). Each strategy was introduced and practiced throughout the session. Between the sessions, the participants were given homework to exercise the strategies (9 homework tasks in total). Frequency of attendance and frequency of completed homework tasks were noted.

Comparable to a similar cognitive intervention study in MCI [23], we did not include an active control group, as the aim of the study was the prediction of the individual response rather than evaluating the effectiveness of the training per se. To quantify the effect of the intervention (without aiming to separate effects of the specific content from those resulting non-specifically from weekly meetings), we compared the results of our study to patients with MCI from both the Alzheimer's Disease Neuroimaging Initiative (ADNI) database ${ }^{1}(n=302$; Table 1) and the local memory clinic $(n=20$; Table 1$)$. Both control samples did not receive cognitive intervention. As the very unequal sample sizes affected the homogeneity of variance assumption (e.g., $\mathrm{F}_{(2,344)}=7.31$, $p<0.001$ for verbal delayed recall), only data of the local memory clinic were statistically compared to the intervention group (see below), while data from ADNI are reported descriptively.

\footnotetext{
${ }^{1}$ Data used in the preparation of this article were in part obtained from the Alzheimer's Disease Neuroimaging Initiative (ADNI) database (adni.loni.usc.edu). The ADNI was launched in 2003 as a public-private partnership, led by Principal Investigator Michael W. Weiner, MD. The primary goal of ADNI has been to test whether serial magnetic resonance imaging (MRI), positron emission tomography (PET), other biological markers, and clinical and neuropsychological assessment can be combined to measure the progression of mild cognitive impairment (MCI) and early Alzheimer's disease (AD). For up-to-date information, see www.adni-info.org.
} 
Table 1

Subject characterization for the cognitive intervention group, the ADNI study, and data from the local memory clinic. The latter two groups did not receive a cognitive intervention. Please note that in the cognitive intervention group 'time to first follow-up' represents the time between pre- to post-testing

\begin{tabular}{|c|c|c|c|c|c|c|c|}
\hline & \multicolumn{2}{|c|}{ Cognitive intervention } & \multicolumn{2}{|c|}{ ADNI* } & \multicolumn{2}{|c|}{ Local memory clinic } & \multirow[t]{2}{*}{$p$-value } \\
\hline & Mean & SD & Mean & $\mathrm{SD}$ & Mean & $\mathrm{SD}$ & \\
\hline $\mathrm{n}(\mathrm{M} / \mathrm{F})$ & $25(14 / 11)$ & & $302(190 / 112)$ & & $20(10 / 10)$ & & \\
\hline Age $(y)$ & 72.9 & 5.1 & 74.1 & 7.1 & 72.9 & 6.1 & 0.99 \\
\hline Education (y) & 13.6 & 3.2 & 15.7 & 3.0 & 12.0 & 3.9 & 0.13 \\
\hline Time to first follow-up (mo) & 6.2 & 2.8 & 6.0 & / & 7.5 & 3.1 & 0.18 \\
\hline Time to second follow-up (mo) & 11.2 & 2.9 & 12.0 & l & 14.2 & 5.2 & 0.01 \\
\hline MMSE (baseline) & 25.5 & 2.2 & 27.0 & 1.8 & 25.3 & 2.2 & 0.77 \\
\hline Verbal delayed recall (baseline) & 3.5 & 1.6 & 2.7 & 3.2 & 3.3 & 1.7 & 0.65 \\
\hline
\end{tabular}

ADNI, Alzheimer's disease Neuroimaging Initiative; SD, standard deviation; MMSE, Mini-Mental Status Examination. *Sociodemographic variables were only compared between the local memory group and the cognitive intervention group.

\section{Magnetic resonance imaging}

Structural MRI was acquired at baseline (i.e., pre-training) using a three-dimensional Magnetization Prepared Rapid Acquisition Gradient Echo (MPRAGE) sequence and a 12-channel standard head coil in a $3 \mathrm{~T}$ scanner (Tim Trio; Siemens, Erlangen, Germany). The parameters applied were as follows: flip angle of $12^{\circ}$, repetition time (TR) of $2200 \mathrm{~ms}$, echo time (TE) of $2.15 \mathrm{~s}$, matrix size of $256 \times 256 \times 176$, and slice thickness of $1 \mathrm{~mm}$.

\section{Hippocampal and entorhinal cortex volumetry}

Structural MRI was automatically processed using FreeSurfer 5.3 [37] to obtain gray matter volumes of the left and right entorhinal cortices. Volumes of the left and right hippocampi and the respective subfields were automatically segmented using FreeSurfer 6.0 $\operatorname{dev}$ [38] (http://surfer.nmr.mgh.harvard.edu), as it uses a second modality and was assumed to produce more accurate estimates of hippocampal subfield volumes [39]. FreeSurfer segmentations were qualitatively checked by two raters and compared to an anatomical atlas. An agreement between raters was sought on all cases with low segmentation quality.

Normalized entorhinal cortex and hippocampal volumes (and the respective subfields) were calculated for each individual, using residuals from a linear regression between volume of the respective region (y) and total intracranial volume (x) as described by Jack et al. [40].

\section{Statistical analyses}

Statistical analyses were performed using SPSS (version 21.0; IBM Inc.; Armonk, USA), parametric tests (Shapiro-Wilk's tests did not indicate that the assumption of normality was violated), and a $p$-value below 0.05 (uncorrected for multiple comparisons) for statistical significance.

\section{Cognitive change after intervention}

A repeated measures ANOVA was used to test for significant differences between pre- and post-training in verbal delayed recall of the CERAD wordlist, global cognition (MMSE), and speed of information processing, using group as between-subjects factor and time as within-subject factor (i.e., pre-training, post-training and follow-up for the cognitive intervention group or baseline, 6-months and 12-months follow-up for the no-intervention group).

A repeated measures ANOVA was also used for spatial navigation with time as within-subject factor. Note that we were not able to compare performance in this task to the local no-intervention group (and to the ADNI control sample) as the here applied RLT task was not included in either neuropsychological assessment.

\section{Predicting response to cognitive training}

For an actual implementation in the clinical routine, we first predicted the response to cognitive training simply based on age and cognitive performance at baseline (i.e., simple model). Subsequently, we quantified the improvement of predictive accuracy when adding MRI-based biological variables (e.g., hippocampal volume), gene-based biological variables (i.e., APOE), and motivation as additional regressors.

Response predictions were tested using stepwise linear regressions (probability of $\mathrm{F}$ for entry: $p \leq 0.05$, probability of $\mathrm{F}$ for removal: $p \geq 0.10$ ) 
and the change between pre- and post-training as dependent variables. In stepwise linear regression, independent variables are included one at a time in successive order of incremental predictive value (i.e., the predictor explaining the most variance is entered first). For verbal delayed recall, volume of the left hippocampus and the left entorhinal cortex were used as independent variables. The analysis was restricted to the left hemisphere, where verbal information is primarily processed $[24,25]$. For spatial navigation, which is primarily processed in the right hemisphere, volume of the right hippocampal tail (i.e., the posterior hippocampus) and the right pre-subiculum [27] were used as independent variables. For the MMSE, volumes of either left or right hippocampus [41] and left or right entorhinal cortex [42] were used as independent variables (i.e., one linear regression was computed for the left- and another one for the right hemisphere). In case of a significant prediction of verbal delayed recall (or the MMSE, respectively) from volume of the (whole) hippocampus, further associations were computed with its' subfields. As speed of information processing is usually not improved by strategy training (although cognitive exercises can be somewhat beneficial [5]), we did not predict change between pre- and post-training for this variable. Presence of APOE $\varepsilon 4$ alleles and frequency of completed homework task were included in all regression analyses. As a high number of participants (88\%) attended the complete cognitive intervention, we did not include frequency of attendance in the prediction models.

\section{RESULTS}

Data were available from 21 participants directly at post-training and from 19 participants 6-months later (Table 2). Attendance rates were high: $88 \%$ of the participants completed all training sessions, the remaining participants completed five $(n=2)$ or four $(n=1)$ sessions, respectively. On average, 6.5 (out of 9) homework tasks were completed (SD 2.1). Figure 2 depicts the variability within the response to cognitive training. Three patients with MCI were APOE $\varepsilon 4$ homozygotes, 12 were APOE $\varepsilon 4$ heterozygotes and 10 had no APOE $\varepsilon 4$ allele.

\section{Cognitive change after intervention}

We found a significant interaction between group (intervention versus no-intervention) and time $\left(\mathrm{F}_{(2,72)}=3.82, p<0.05\right)$ for verbal delayed recall. Patients with MCI in the cognitive training group slightly increased their verbal delayed recall over time, while patients with MCI from the local memory clinic, who did not receive cognitive intervention, significantly declined during the same time interval (Fig. 3). We also found a significant interaction between group and time for the MMSE $\left(\mathrm{F}_{(2,72)}=8.39, p<0.001\right)$. Patients with MCI in the cognitive training group increased their MMSE scores, while those from the local memory clinic decreased during the same time interval (Fig. 3). For the TMT-A, we did not find a significant interaction between group and time $\left(\mathrm{F}_{(2,72)}=0.07, p=0.93\right)$. Both patients with MCI in the cognitive training group and in the external comparison group required almost the same time to complete the TMT-A as compared to baseline (Supplementary Figure 1). Spatial navigation significantly improved after the cognitive intervention $\left(\mathrm{F}_{(1,36)}=8.56, p<0.001\right)$ (Fig. 4). We did not find any significant difference between MCI subtypes regarding change in verbal delayed recall, global cognition, spatial navigation, or speed of information processing (all $p>0.3$ ).

\section{Predicting response to cognitive training}

Results of stepwise linear regressions are detailed in Table 3. The simple model, representing the

Table 2

Pre- and post-training scores for the cognitive intervention group

\begin{tabular}{|c|c|c|c|c|c|c|}
\hline & \multicolumn{2}{|c|}{$\begin{array}{l}\text { pre-training } \\
(n=25)\end{array}$} & \multicolumn{2}{|c|}{$\begin{array}{c}\text { post-training } \\
(n=21)\end{array}$} & \multicolumn{2}{|c|}{$\begin{array}{c}\text { 6-months follow-up } \\
(n=19)\end{array}$} \\
\hline & Mean & SD & Mean & SD & Mean & SD \\
\hline MMSE (0-30) & 25.5 & 2.2 & 26.4 & 1.7 & 27.1 & 2.1 \\
\hline Verbal delayed recall (0-10) & 3.5 & 1.6 & 3.6 & 2.5 & 3.7 & 1.9 \\
\hline Route learning task errors (0-9) & 2.4 & 1.4 & 1.0 & 0.9 & 1.6 & 1.3 \\
\hline Processing speed (0-180 s) & 52.4 & 19.4 & 47.1 & 18.3 & 51.5 & 15.9 \\
\hline Depressive symptoms $(0-63)$ & 9.6 & 6.6 & 9.4 & 6.9 & 8.8 & 4.8 \\
\hline
\end{tabular}

SD, standard deviation; MMSE, Mini-Mental Status Examination; depressive symptoms were assessed with the Beck's Depression Inventory II. 

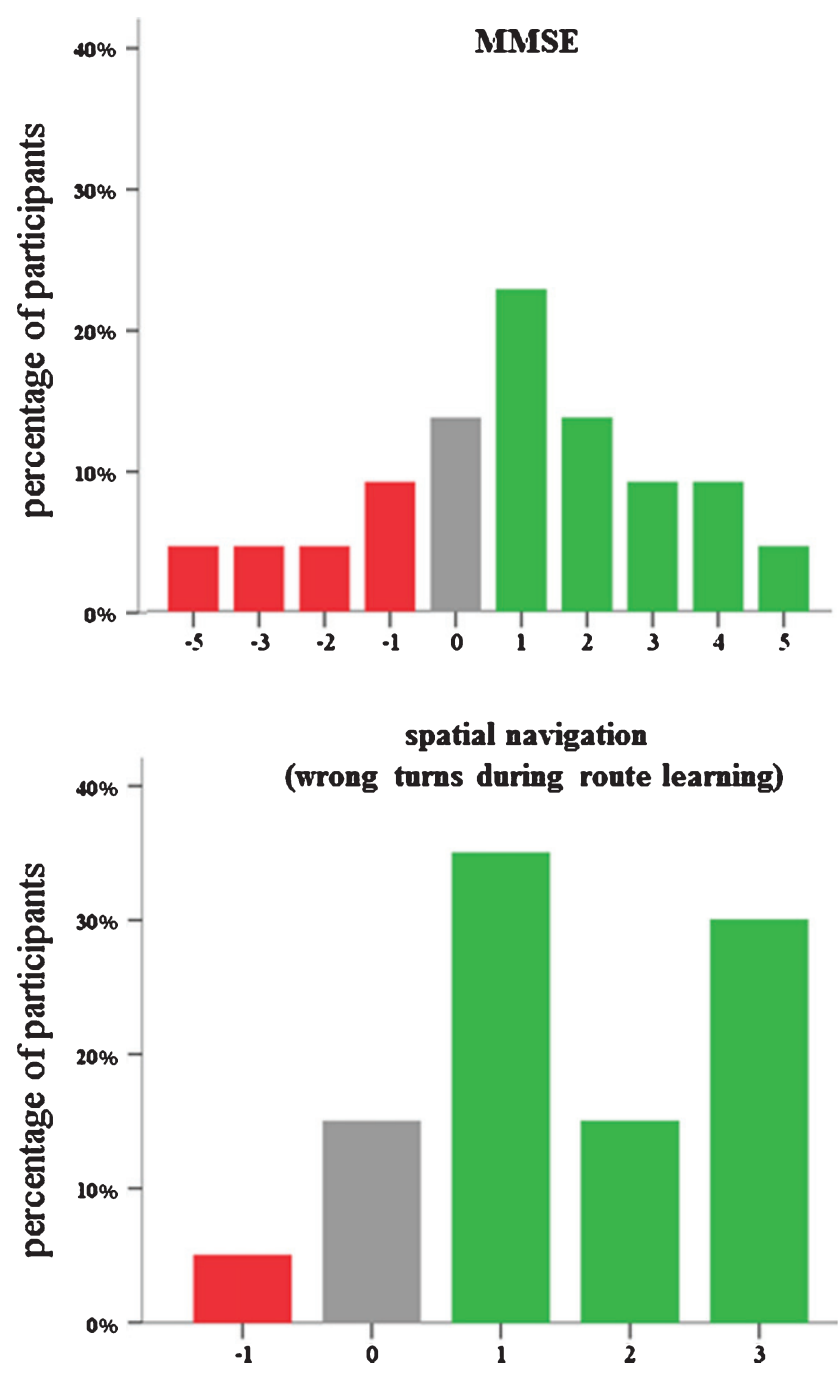

verbal delayed recall
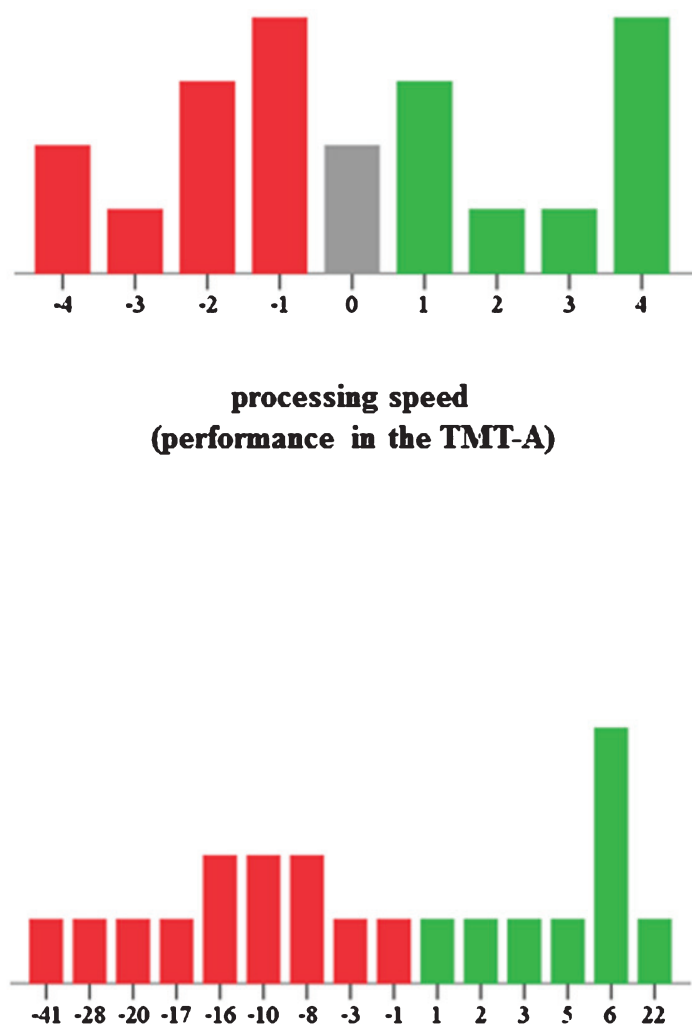

Fig. 2. Variability in the response to cognitive training for the Mini-Mental Status Examination (MMSE), verbal delayed recall, spatial navigation, and processing speed in patients with mild cognitive impairment. Negative scores (red color) indicate decline from pre- to post-training, while positive scores (green color) depict an improvement. Note: For the TMT-A and spatial navigation, scores were recoded such that positive scores indicate an improvement, too.

clinical setting, yielded no significant result in predicting the increase in verbal delayed recall (i.e., neither age nor baseline performance explained a significant proportion of the improvement after training). When adding the MRI-based biological variables, the gene-based biological variables, and motivation as additional regressors, verbal delayed recall improvement was significantly predicted by volume of the left entorhinal cortex and frequency of completed homework, with more volume and more frequently completed homework being associated with greater increase in verbal delayed recall (Table 3, Fig. 5). Left hippocampal volume, age, and baseline performance did not explain significantly more variance in the prediction model (Supplementary Table 2; see also Supplementary Table 4 for a correlation matrix).

For the increase in spatial navigation and the MMSE, only baseline performance was a significant predictor in both the simple model (Supplementary Table 3) and the full model (Table 3).

The presence of one or two APOE alleles was no significant predictor of any of the cognitive change variables (Supplementary Tables) and frequency of completed homework tasks only predicted change in verbal delayed recall (Supplementary Table 2). 

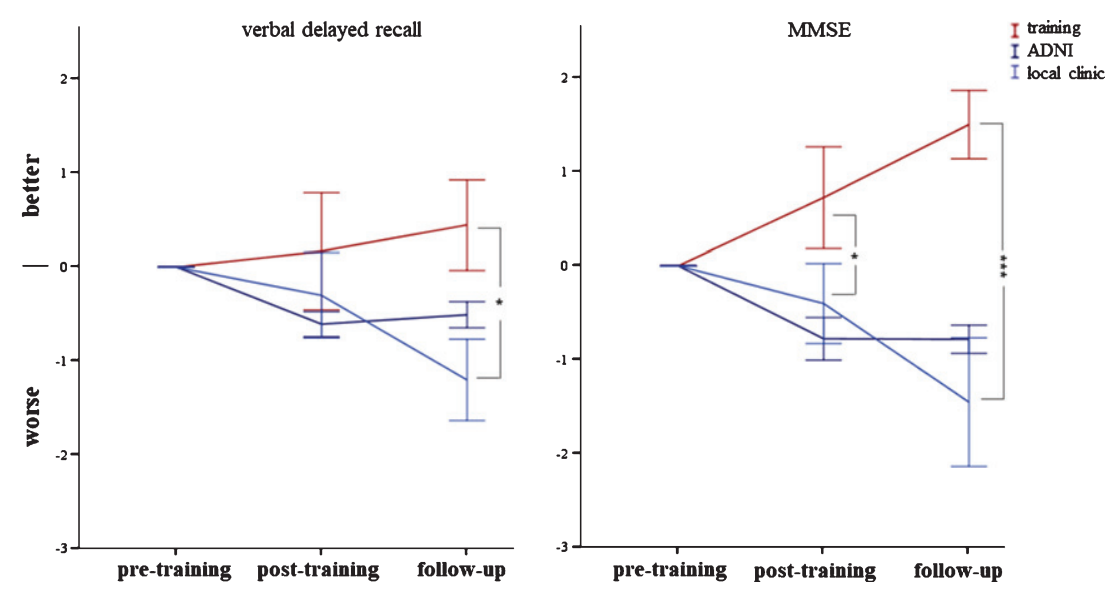

Fig. 3. Change in verbal delayed recall and Mini-Mental Status Examination (MMSE) between pre- to post-training and 6 months later. The cognitive intervention group is shown in red, the ADNI and local memory clinic are depicted in blue (the latter two did not receive a cognitive training). Significant at $* p<0.05$ or $* * * p<0.001$, respectively. Error bars indicate \pm 1 standard error of the mean. Note: Data from the ADNI was not included in the statistical analysis.

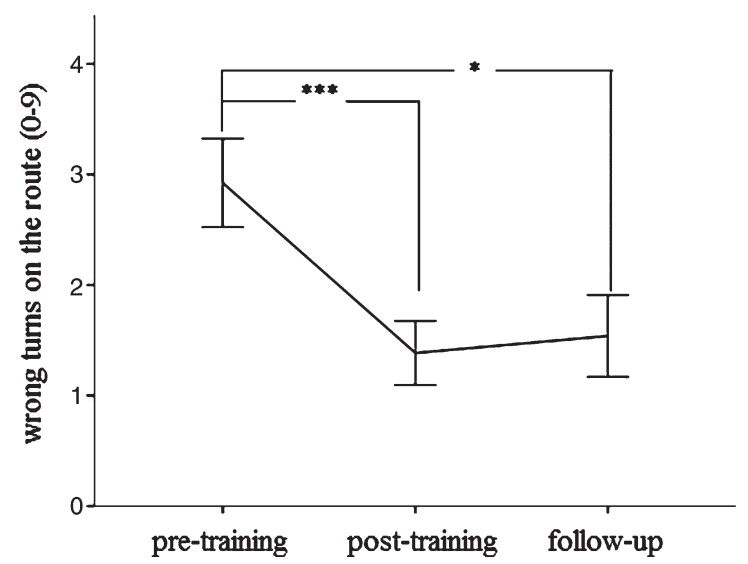

Fig. 4. Change in spatial navigation (route learning task) between pre- to post-training and 6 months later. Significant at $* p<0.05$ or $* * * p<0.001$, respectively. Error bars indicate \pm 1 standard error of the mean.

\section{DISCUSSION}

The aim of this study was to analyze the predictive value of MRI- and gene-based biological factors when patients with MCI receive a six-week mnemonic strategy training. Rather than comparing an intervention group against a control condition, our aim was to identify markers that differentiate those who benefit from cognitive intervention from those who do not. For the outcome after training, we examined verbal delayed recall as a primary target and the MMSE, spatial navigation, and speed of information processing to study near and far transfer effects. We also measured longitudinal effects of the intervention, as these are clinically relevant.

After strategy training (and at follow-up, too), we found a slight improvement in verbal delayed recall scores and an increase in the MMSE, while patients with MCI who did not receive cognitive training significantly declined in both tasks (Fig. 3). It is unlikely that the increase in cognitive performance in the intervention group is due to the repeated completion of the cognitive tests (i.e., re-test effect), as the sample of patients with MCI from the local memory clinic (and the ADNI study) decreased in both tasks, when tested at the same time intervals with identical (or comparable) cognitive tests. Although we did not include an active control group that would have linked the increase in cognition specifically to mnemonic

Table 3

Results of stepwise linear regressions with change after cognitive intervention as outcome variables, different brain areas, frequency of completed homework tasks, age, and baseline performance as predictors

\begin{tabular}{llcrc}
\hline Change between pre- and post-training & predictor variable & $r^{2}$ & $\beta$ & $p$ \\
\hline Verbal delayed recall & left entorhinal cortex volume & 0.52 & 0.47 & 0.01 \\
& frequency of completed homework & & 0.44 & 0.02 \\
Mini Mental Status Examination & baseline performance & 0.69 & -0.84 & $<0.001$ \\
Spatial navigation (route learning) & baseline performance & 0.49 & -0.72 & 0.001 \\
\hline
\end{tabular}

Note: Only significant results are depicted. 


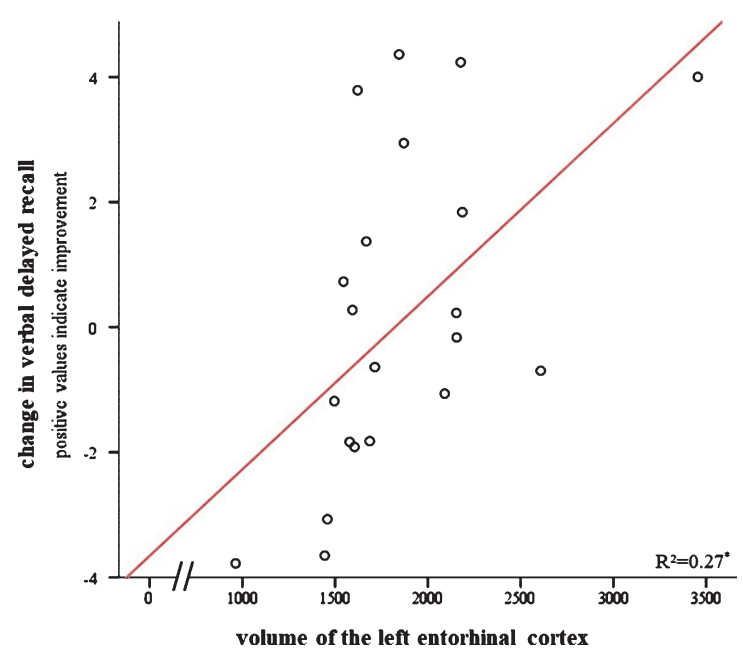

Fig. 5. Relationship between change in verbal delayed recall after cognitive training (i.e., pre- to post-intervention) and volume of the left entorhinal cortex. Positive scores on the $y$-axis indicate improvement. Significant at $* p<0.05$.

strategy training, our results support previous findings of strategy training to lead to a stabilization of verbal delayed recall despite a progressing neurodegenerative disorder $[10,11]$.

We did not find any effect of the cognitive intervention on processing speed, as performance remained comparable to the two external data sets (Supplementary Figure 1). Finally, we found a highly significant improvement in the RLT after the cognitive intervention (Fig. 4). Although the use of alternative routes makes simple re-tests effects less likely, we cannot exclude habituation effects. The RLT may be particularly prone to such effects, as being chauffeured in a wheelchair may need adaption to this unfamiliar situation. Alternatively, cognitive training may indeed generalize across both hippocampi, or, more generally, the knowledge of the possibility to use a strategy for episodic memory enhancement can lead to the use of similar strategies during other learning-dependent tasks.

For the prediction of the trainings response with MRI- and gene-based biological factors, we found that patients with larger left entorhinal cortex volume improved significantly more in verbal delayed recall after cognitive training (Fig. 6). On the contrary, disease-specific biological factors did not significantly improve the predictive accuracy for the RLT and the MMSE. This is most likely explained by ceiling effects and the low sensitivity of the MMSE in the MCI stage [29]. The interpretation is supported by a visual display of the data showing that only those individuals with initially lower scores improved after the cognitive intervention (Supplementary Figure 2). For verbal delayed recall, a test without ceiling in the elderly population, we found a significantly positive relationship between volume of the entorhinal cortex and the increase in verbal delayed recall. We found no such relationship with volume of the left hippocampus reported previously for individuals with SMI [14]. Different segmentation and parcellation algorithms (FreeSurfer 5.3 versus 6.0, different imaging data (T1 versus T1 and FLAIR), and different population criteria may contribute to the disparate findings. For both SMI and MCI, the entorhinal cortex deteriorates more heavily than the hippocampus (volume reduction entorhinal cortex 18\% in SMI and $26 \%$ in MCI; volume reduction hippocampus $6 \%$ in SMI and 16\% in MCI; $[43,44])$. Thus it seems that the entorhinal cortex is an important area for both groups although in patients with MCI it might deteriorate more rapidly than in individuals with SMI. Regarding the discrepancy between our findings and that of Engvig et al. [14], we note that these authors did not include entorhinal cortex volume in their analysis which makes a direct comparison difficult. However, in a more recent paper of this group [45] they showed that grey matter volume of the left entorhinal cortex increased similarly after cognitive training for both healthy controls and patients with SMI while no such effect was found for the hippocampus. Thus, there seems to be an association between cognitive intervention and the entorhinal cortex in both SMI and MCI. Hence, both areas might differentially shrink over the course of AD but in a similar way in both SMI and MCI.

Motivation of the participants (as indexed by frequency of completed homework tasks) was also a significant predictor of improvement in verbal delayed recall. In cognitive behavioral therapy, homework non-compliance is one of the top most reason for therapy failure and there is a strong association between patients' motivation and homework completion as well as between homework completion and drop-out rate or therapy outcome [47]. Interestingly, when examining the number of completed homework tasks for drop-outs and completers of the intervention, we found that those who did not complete the intervention far less often completed their homework tasks (drop-outs: $3.7 \pm 2.6$; completers: $7.8 \pm 1.7 ; \mathrm{t}(23)=4.65, p<0.001)$, which remained significant when controlling for attendance rates $\left(\mathrm{F}_{(2,22)}=4.49 ; p<0.05\right)$. Therefore, we believe that homework should be an essential part of cognitive 


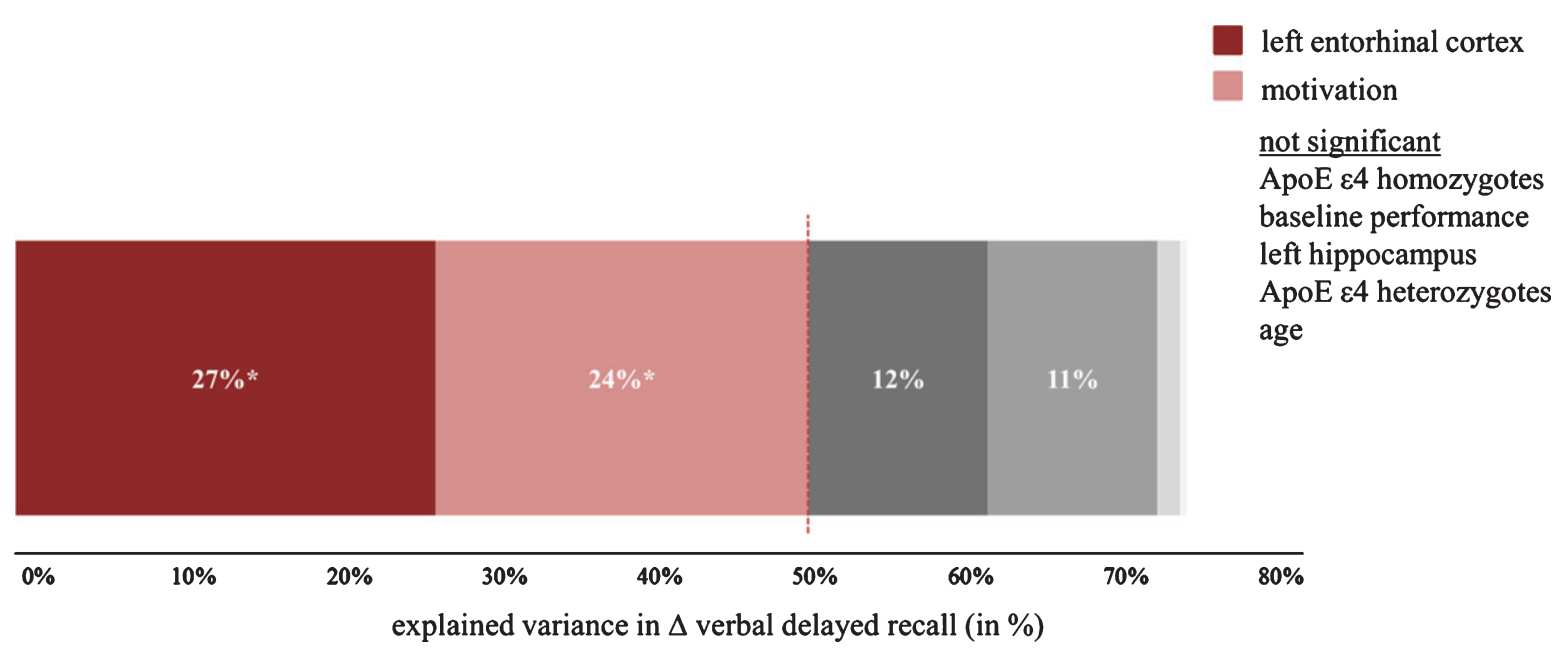

Fig. 6. Explained variance for the prediction of verbal delayed recall change after cognitive training (i.e., pre- to post-intervention). Significant predictors are depicted in different shades of red, while grey color indicates a non-significant predictor. Please note that some non-significant predictors are invisible due to a very small amount of explained variance.

interventions. Future studies might include questionnaires on motivation (and perhaps personality traits) in order to replicate our results and to investigate the underlying cause of this relationship. It could well be that motivation reflects personality traits; on the other hand, external factors such as effort, amount of time consumed, or understanding the purpose of the homework might also play a role. The fact that motivation did not significantly explain performance changes in the MMSE and the RLT is explained again by ceiling effects in those tasks. Importantly, age was no significant predictor for interventionrelated changes in verbal delayed recall. This finding is contrary to a previous study by Belleville et al. who found younger age and more years of education to be significantly correlated with an increase in episodic memory after memory training. We did neither find such a correlation for age nor education in our study $(p>0.4)$. However, we believe the study by Belleville and colleagues is not directly comparable to our study. Most importantly, they included both healthy controls and patients with MCI and they did not report if the correlations were significant in both groups. Second, their intervention group was younger ( $62.3 \pm 7.3)$ than our intervention group (age: $73.4 \pm 5.3$ ). Third, their patients with MCI had a very high MMSE score $(28.9 \pm 1.2)$ —which was very similar to that of their healthy controls $(29.0 \pm 0.8)$-while in our study the mean MMSE score was $25.5( \pm 2.2)$. Besides age, baseline performance did also not significantly predict the change in verbal delayed recall, demonstrating the clinical usefulness of biological, disease-specific predictor variables (Fig. 6). In practice, cognitive training should be recommended particularly to individuals with a bigger entorhinal cortex as individuals who did not benefit from the intervention showed a $17 \%$ smaller entorhinal cortex volume compared to those who increased their verbal delayed recall scores (no improvement: $1675 \pm 429 \mathrm{~mm}^{3}$; improvement: $2011 \pm 498 \mathrm{~mm}^{3}$ ). This difference is significant at $p<0.05$. A post-hoc ROC analysis also revealed a significant result (area under the curve $=0.76[95 \%$ CI, $0.53-0.98], p<0.05)$ and an optimal cut-off derived from the ROC analysis at $1659.7 \mathrm{~mm}^{3}$ with a sensitivity and specificity of 0.7 .

\section{Limitation}

The results of our study might be limited by the relatively small sample size $(n=25)$ although other cognitive intervention studies recruited even fewer participants $(n<20$, e.g., $[12,14])$. When using a bootstrapping technique [46] to evaluate the stability of our key finding, the same variables became significant in $72 \%$ (volume of the left entorhinal cortex) and 85\% (motivation) of all 1000 subsamples (Supplementary Table 5). Variables that are selected to be significant in $50 \%$ or more of the data sets are considered to have a stable association with the outcome [46]. Bootstrapping also reduces the likelihood of Type I errors and increases the likelihood of out of sample replication [46].

None of our predictors became significant when the change between baseline and follow-up was used as a dependent variable. We conclude that too many 
external/behavioral factors contribute to the longterm effects.

Besides absolute change between pre- and posttraining, percent change might also serve as a marker of improvement. However, we decided to focus on the change in the absolute number of remembered words rather than the proportional change. In our view, an improvement of, for example, two words at an initially high level is no less significant in terms of cognition than an improvement at a lower baseline performance although we realize that the consequences for the individual might differ.

\section{Conclusion}

In summary, we found cognitive training to stabilize or even improve learning-dependent behavior. Stratifying patients with MCI based on diseasespecific biological factors might be a useful step towards individualized medicine, when extended to a range of alternative treatment options.

\section{ACKNOWLEDGMENTS}

JP was funded from the University Medical Centre Freiburg.

Data collection and sharing for the ADNI project was funded by the Alzheimer's Disease Neuroimaging Initiative (ADNI) (National Institutes of Health Grant U01 AG024904) and DOD ADNI (Department of Defense award number W81XWH-12-2-0012). ADNI is funded by the National Institute on Aging, the National Institute of Biomedical Imaging and Bioengineering, and through generous contributions from the following: AbbVie, Alzheimer's Association; Alzheimer's Drug Discovery Foundation; Araclon Biotech; BioClinica, Inc.; Biogen; BristolMyers Squibb Company; CereSpir, Inc.; Cogstate; Eisai Inc.; Elan Pharmaceuticals, Inc.; Eli Lilly and Company; EuroImmun; F. Hoffmann-La Roche Ltd and its affiliated company Genentech, Inc.; Fujirebio; GE Healthcare; IXICO Ltd.; Janssen Alzheimer Immunotherapy Research \& Development, LLC.; Johnson \& Johnson Pharmaceutical Research \& Development LLC.; Lumosity; Lundbeck; Merck \& Co., Inc.; Meso Scale Diagnostics, LLC.; NeuroRx Research; Neurotrack Technologies; Novartis Pharmaceuticals Corporation; Pfizer Inc.; Piramal Imaging; Servier; Takeda Pharmaceutical Company; and Transition Therapeutics. The Canadian Institutes of Health Research is providing funds to support ADNI clinical sites in Canada. Private sector con- tributions are facilitated by the Foundation for the National Institutes of Health (http://www.fnih.org). The grantee organization is the Northern California Institute for Research and Education, and the study is coordinated by the Alzheimer's Therapeutic Research Institute at the University of Southern California. ADNI data are disseminated by the Laboratory for Neuro Imaging at the University of Southern California.

Authors' disclosures available online (http://j-alz. com/manuscript-disclosures/17-0580r2).

\section{SUPPLEMENTARY MATERIAL}

The supplementary material is available in the electronic version of this article: http://dx.doi.org/ 10.3233/JAD-170580.

\section{REFERENCES}

[1] Winblad B, Gauthier S, Scinto L, Feldman H, Wilcock GK, Truyen L, Mayorga AJ, Wang D, Brashear HR, Nye JS, GAL-INT-11/18 Study Group (2008) Safety and efficacy of galantamine in subjects with mild cognitive impairment. Neurology 70, 2024-2035.

[2] Cooper C, Li R, Lyketsos C, Livingston G (2013) Treatment for mild cognitive impairment: Systematic review. $\mathrm{Br}$ J Psychiatry J Ment Sci 203, 255-264.

[3] Russ TC, Morling JR (2012) Cholinesterase inhibitors for mild cognitive impairment. Cochrane Database Syst Rev, CD009132.

[4] Vemuri P, Fields J, Peter J, Klöppel S (2016) Cognitive interventions in Alzheimer's and Parkinson's diseases: Emerging mechanisms and role of imaging. Curr Opin Neurol 29, 405-411.

[5] Jean L, Bergeron M-E, Thivierge S, Simard M (2010) Cognitive intervention programs for individuals with mild cognitive impairment: Systematic review of the literature. Am J Geriatr Psychiatry 18, 281-296.

[6] Li H, Li J, Li N, Li B, Wang P, Zhou T (2011) Cognitive intervention for persons with mild cognitive impairment: A meta-analysis. Ageing Res Rev 10, 285-296.

[7] Gates NJ, Sachdev PS, Fiatarone Singh MA, Valenzuela M (2011) Cognitive and memory training in adults at risk of dementia: A systematic review. BMC Geriatr 11, 55.

[8] Noack H, Lövdén M, Schmiedek F, Lindenberger U (2009) Cognitive plasticity in adulthood and old age: Gauging the generality of cognitive intervention effects. Restor Neurol Neurosci 27, 435-453.

[9] Folstein MF, Folstein SE, McHugh PR (1975) "Minimental state". A practical method for grading the cognitive state of patients for the clinician. J Psychiatr Res 12, 189-198.

[10] Rapp S, Brenes G, Marsh AP (2002) Memory enhancement training for older adults with mild cognitive impairment: A preliminary study. Aging Ment Health 6, 5-11.

[11] Troyer AK, Murphy KJ, Anderson ND, Moscovitch M, Craik FIM (2008) Changing everyday memory behaviour in amnestic mild cognitive impairment: A randomised controlled trial. Neuropsychol Rehabil 18, 65-88. 
[12] Londos E, Boschian K, Lindén A, Persson C, Minthon L, Lexell J (2008) Effects of a goal-oriented rehabilitation program in mild cognitive impairment: A pilot study. Am $J$ Alzheimers Dis Other Demen 23, 177-183.

[13] Buschert VC, Giegling I, Teipel SJ, Jolk S, Hampel H, Rujescu D, Buerger K (2012) Long-term observation of a multicomponent cognitive intervention in mild cognitive impairment. J Clin Psychiatry 73, e1492-e1498.

[14] Engvig A, Fjell AM, Westlye LT, Skaane NV, Sundseth Ø, Walhovd KB (2012) Hippocampal subfield volumes correlate with memory training benefit in subjective memory impairment. Neuroimage 61, 188-194.

[15] Brooks JO, Friedman L, Pearman AM, Gray C, Yesavage JA (1999) Mnemonic training in older adults: Effects of age, length of training, and type of cognitive pretraining. Int Psychogeriatr IPA 11, 75-84.

[16] Singer T, Lindenberger U, Baltes PB (2003) Plasticity of memory for new learning in very old age: A story of major loss? Psychol Aging 18, 306-317.

[17] Belleville S, Gilbert B, Fontaine F, Gagnon L, Ménard É, Gauthier S (2006) Improvement of episodic memory in persons with mild cognitive impairment and healthy older adults: Evidence from a cognitive intervention program. Dement Geriatr Cogn Disord 22, 486-499.

[18] Teipel SJ, Cavedo E, Grothe MJ, Lista S, Galluzzi S, Colliot O, Chupin M, Bakardjian H, Dormont D, Dubois B, Hampel H, Hippocampus Study, Group (2016) Predictors of cognitive decline and treatment response in a clinical trial on suspected prodromal Alzheimer's disease. Neuropharmacology 108, 128-135.

[19] Petersen RC, Thomas RG, Grundman M, Bennett D, Doody R, Ferris S, Galasko D, Jin S, Kaye J, Levey A, Pfeiffer E, Sano M, van Dyck CH, Thal LJ, Alzheimer's Disease Cooperative Study, Group (2005) Vitamin E and donepezil for the treatment of mild cognitive impairment. $N$ Engl $J$ Med 352, 2379-2388.

[20] Small SA, Schobel SA, Buxton RB, Witter MP, Barnes CA (2011) A pathophysiological framework of hippocampal dysfunction in ageing and disease. Nat Rev Neurosci 12, 585-601.

[21] Zhou M, Zhang F, Zhao L, Qian J, Dong C (2016) Entorhinal cortex: A good biomarker of mild cognitive impairment and mild Alzheimer's disease. Rev Neurosci 27, 185-195.

[22] Rosen AC, Prull MW, Gabrieli JDE, Stoub T, O'Hara R, Friedman L, Yesavage JA, deToledo-Morrell L (2003) Differential associations between entorhinal and hippocampal volumes and memory performance in older adults. Behav Neurosci 117, 1150-1160.

[23] Finauer G, ed (2007) Therapiemanuale für die neuropsychologische Rehabilitation: Kognitive und kompetenzorientierte Therapie für die Gruppen- und Einzelbehandlung, Springer Medizin, Heidelberg.

[24] Belleville S, Clément F, Mellah S, Gilbert B, Fontaine F, Gauthier S (2011) Training-related brain plasticity in subjects at risk of developing Alzheimer's disease. Brain $J$ Neurol 134, 1623-1634.

[25] Chen KHM, Chuah LYM, Sim SKY, Chee MWL (2010) Hippocampal region-specific contributions to memory performance in normal elderly. Brain Cogn 72, 400-407.

[26] Bonner-Jackson A, Mahmoud S, Miller J, Banks SJ (2015) Verbal and non-verbal memory and hippocampal volumes in a memory clinic population. Alzheimers Res Ther 7, 61 .

[27] Wehling E, Lundervold AJ, Standnes B, Gjerstad L, Reinvang I (2007) APOE status and its association to learning and memory performance in middle aged and older
Norwegians seeking assessment for memory deficits. Behav Brain Funct 3, 57.

[28] Wolbers T, Dudchenko PA, Wood ER (2014) Spatial memory-a unique window into healthy and pathological aging. Front Aging Neurosci 6, 35.

[29] Kessels RPC, de Haan EHF, Kappelle LJ, Postma A (2001) Varieties of human spatial memory: A meta-analysis on the effects of hippocampal lesions. Brain Res Rev 35, 295-303.

[30] Nasreddine ZS, Phillips NA, Bédirian V, Charbonneau S, Whitehead V, Collin I, Cummings JL, Chertkow H (2005) The Montreal Cognitive Assessment, MoCA: A brief screening tool for mild cognitive impairment. J Am Geriatr Soc 53, 695-699.

[31] Morris JC, Heyman A, Mohs RC, Hughes JP, van Belle G, Fillenbaum G, Mellits ED, Clark C (1989) The Consortium to Establish a Registry for Alzheimer's Disease (CERAD). Part I. Clinical and neuropsychological assessment of Alzheimer's disease. Neurology 39, 1159-1165.

[32] Petersen RC, Smith GE, Waring SC, Ivnik RJ, Tangalos EG, Kokmen E (1999) Mild cognitive impairment: Clinical characterization and outcome. Arch Neurol 56, 303-308.

[33] Albert MS, DeKosky ST, Dickson D, Dubois B, Feldman HH, Fox NC, Gamst A, Holtzman DM, Jagust WJ, Petersen RC, Snyder PJ, Carrillo MC, Thies B, Phelps CH (2011) The diagnosis of mild cognitive impairment due to Alzheimer's disease: Recommendations from the National Institute on Aging-Alzheimer's Association workgroups on diagnostic guidelines for Alzheimer's disease. Alzheimers Dement 7, 270-279.

[34] Beck AT, Steer RA, Brown GK (1996) BDI-II, Beck depression inventory: Manual, Psychological Corp.; Harcourt Brace, San Antonio, TX; Boston.

[35] Yesavage JA, Sheikh JI (1986) 9/Geriatric Depression Scale (GDS). Clin Gerontol 5, 165-173.

[36] Fazekas F, Chawluk JB, Alavi A, Hurtig HI, Zimmerman RA (1987) MR signal abnormalities at $1.5 \mathrm{~T}$ in Alzheimer's dementia and normal aging. AJR Am J Roentgenol 149, 351356.

[37] deIpolyi AR, Rankin KP, Mucke L, Miller BL, GornoTempini ML (2007) Spatial cognition and the human navigation network in AD and MCI. Neurology 69, 986-997.

[38] Fischl B (2012) FreeSurfer. Neuroimage 62, 774-781.

[39] Iglesias JE, Augustinack JC, Nguyen K, Player CM, Player A, Wright M, Roy N, Frosch MP, McKee AC, Wald LL, Fischl B, Van Leemput K (2015) A computational atlas of the hippocampal formation using ex vivo, ultra-high resolution MRI: Application to adaptive segmentation of in vivo MRI. Neuroimage 115, 117-137.

[40] Whelan CD, Hibar DP, van Velzen LS, Zannas AS, CarrilloRoa T, McMahon K, Prasad G, Kelly S, Faskowitz J, deZubiracay G, Iglesias JE, van Erp TGM, Frodl T, Martin NG, Wright MJ, Jahanshad N, Schmaal L, Sämann PG, Thompson PM (2016) Heritability and reliability of automatically segmented human hippocampal formation subregions. Neuroimage 128, 125-137.

[41] Jack CR, Knopman DS, Weigand SD, Wiste HJ, Vemuri P, Lowe V, Kantarci K, Gunter JL, Senjem ML, Ivnik RJ, Roberts RO, Rocca WA, Boeve BF, Petersen RC (2012) An operational approach to National Institute on AgingAlzheimer's Association criteria for preclinical Alzheimer disease. Ann Neurol 71, 765-775.

[42] Peng G-P, Feng Z, He F-P, Chen Z-Q, Liu X-Y, Liu P, Luo B-Y (2015) Correlation of hippocampal volume and cognitive performances in patients with either mild cognitive 
impairment or Alzheimer's disease. CNS Neurosci Ther 21, 15-22.

[43] Velayudhan L, Proitsi P, Westman E, Muehlboeck JS, Mecocci P, Vellas B, Tsolaki M, Kłoszewska I, Soininen H, Spenger C, Hodges A, Powell J, Lovestone S, Simmons A; dNeuroMed Consortium (2013) Entorhinal cortex thickness predicts cognitive decline in Alzheimer's disease. J Alzheimers Dis 33, 755-766.

[44] Jessen F, Feyen L, Freymann K, Tepest R, Maier W, Heun $\mathrm{R}$, Schild H-H, Scheef L (2006) Volume reduction of the entorhinal cortex in subjective memory impairment. Neurobiol Aging 27, 1751-1756.
[45] Engvig A, Fjell AM, Westlye LT, Skaane NV, Dale AM, Holland D, Due-Tønnessen P, Sundseth O, Walhovd KB (2014) Effects of cognitive training on gray matter volumes in memory clinic patients with subjective memory impairment. J Alzheimers Dis 41, 779-791.

[46] Rizopoulos D (2009) bootStepAIC: Bootstrap stepAIC.

[47] Baucom BR, Atkins DC, Rowe LS, Doss BD, Christensen A (2015) Prediction of treatment response at 5-year follow-up in a randomized clinical trial of behaviorally based couple therapies. J Consult Clin Psychol 83, 103-114. 